\title{
Dimensiones de la vía aérea en la población de Cuenca-Ecuador
}

\author{
Airway dimensions in the population of Cuenca-Ecuador \\ Serrano Béjar Leonardo Alfredo', Reinoso Murillo Priscila Victoria', \\ Peñafiel Martínez Nadia Rosalía², Vaca Morocho Andrea Jackeline ${ }^{3}$, \\ Serrano Béjar Mónica Viviana’
}

VOLUMEN 39 | Nº 1 | SEPTIEMBRE 2021

FECHA DE RECEPCIÓN: 25/05/2021 FECHA DE APROBACIÓN: 29/09/2021 FECHA PUBLICACIÓN: 29/09/2021

1. Médico/a en libre ejercicio. Cuenca - Ecuador

2. Hospital Vicente Corral Moscoso Quirófano. Anestesiología. Cuenca - Ecuador

3. Ministerio de Salud Pública. Centro de Salud La Merced. Medicina general.

Cuenca - Ecuador

\section{Artículo Original original Article}

DOI: https://doi.org/10.18537/RFCM.39.01.03

Correspondencia:

lasb91dgmail.com

Dirección:

Mariano Cueva 2032 y Avenida de las Américas

Código Postal:

010101

Celular:

0997320911

Cuenca - Ecuador

\section{RESUMEN}

Objetivo: describir las características de la vía aérea en una población local con la cabeza en posición neutra, según: distancia entre incisivos superiores medios hasta cuerdas vocales, cartílago cricoides, carina y el punto entre el tercer y cuarto anillo traqueal, contado desde la carina en sentido cefálico; cuerdas vocales a carina, tráquea, número de anillos traqueales y correlacionar con estatura y sexo.

Metodología: estudio descriptivo en pacientes intervenidos quirúrgicamente en el Hospital de Especialidades "José Carrasco Arteaga" y Hospital Regional "Vicente Corral Moscoso" durante marzo 2019 a marzo 2020. Se recogieron los datos por medio de un flexómetro, estadímetro y broncoscopio flexible Ambu Scope 3. El análisis de los datos se efectuó en el software IBM-SPSS 22.0 con medidas estadísticas descriptivas y correlaciones.

Resultados: se valoraron 151 pacientes. La longitud de los incisivos medios: a la carina fue de $23.32 \pm(1.98) \mathrm{cm}$, al cricoides de $14.93 \pm(1.38)$ $\mathrm{cm}$, a las cuerdas vocales de $13.07 \pm(1.36) \mathrm{cm}$, al tercer o cuarto anillo traqueal contado desde la carina hacia cefálico $20.81 \pm(2.03) \mathrm{cm}$; de las cuerdas vocales a carina de $10.24 \pm(1.45) \mathrm{cm}$; la tráquea en promedio midió $8.40 \pm(1.37) \mathrm{cm}$, con 13 anillos traqueales.

Conclusión: las medidas de la vía aérea obtenidas están en concordancia con los estudios de poblaciones parecidas a la del estudio, lo cual indica que el extrapolar los datos de los textos clásicos de etnias caucásicas puede ocasionar errores en la intubación endobronquial o extubación inadvertida.

Palabras clave: intubación, manejo de la vía aérea, pacientes internos, anestesia general. 


\section{ABSTRACT}

Objective: to describe the airway characteristics in a local population with the head in a neutral position, according to: distance between upper middle incisors to vocal cords, cricoid cartilage, carina and the point between the third and fourth tracheal ring, counted from the carina cephalad; vocal cords to carina, trachea, number of tracheal rings and correlate with height and sex.

Methodology: it is a descriptive study in patients who were operated at the "Hospital de Especialidades José Carrasco Arteaga" and "Hospital Regional Vicente Corral Moscoso" during March 2019 to March 2020. Data were collected using a flexometer, stadiometer and flexible bronchoscope Ambu Scope 3 The data analysis was carried out in the IBM-SPSS 22.0 software with descriptive statistical measures and correlations.

Results: a total of 151 patients were evaluated. The length of the middle incisors: at the carina was $23.32 \pm(1.98) \mathrm{cm}$, at the cricoid $14.93 \pm(1.38) \mathrm{cm}$, at the vocal cords $13.07 \pm(1.36) \mathrm{cm}$, at the third or fourth tracheal ring counted from the carina towards cephalic $20.81 \pm(2.03) \mathrm{cm}$; from the vocal cords to carina of $10.24 \pm(1.45) \mathrm{cm}$; the trachea on average measured $8.40 \pm(1.37) \mathrm{cm}$, with 13 tracheal rings.

Conclusion: the airway measurements obtained are in agreement with the studies of ethnic groups similar to that of the study population, which indicates that extrapolating the data from the classic texts of Caucasian ethnicities can cause errors in daily practice such as endobronchial intubation or inadvertent extubation.

Key words: intubation, airway management, inpatients, anesthesia general.

\section{INTRODUCCIÓN}

La longitud de la tráquea difiere entre adultos según la etnia, estudios de los años noventa en caucásicos determinaron que la vía aérea mide 29 $\mathrm{cm}$ y varía con un centímetro desde 1.70 metros de altura en promedio por cada $10 \mathrm{~cm}^{1,2}$. Sin embargo, estudios posteriores en países con etnias no caucásicas como Colombia, México e India, indican que hay diferencias en la longitud de la vía aérea en relación con la altura de los pacientes; es decir, que debido a que la población tiene un promedio de altura menor, tendrán en promedio menor longitud de vía aérea ${ }^{3-6}$. No existen datos en el país sobre las medidas de la vía aérea, por ello se extrapolan estudios de otras etnias, usando comúnmente los datos de etnias caucásicas.

La intubación orotraqueal pese a ser un procedimiento que se realiza de forma rutinaria en hospitales, todavía tiene varias contradicciones, incluso entre anestesiólogos experimentados sobre todo el momento de utilizar instrumental y determinar con detalle qué diámetro de tubo usar y a qué profundidad colocarlo, lo cual está relacionado directamente a las medidas de la vía aérea del paciente.

El no tener las medidas en el país predispone a que los pacientes sufran eventos adversos como intubación endobronquial o extubación inadvertida, incluso con personal entrenado. Hay evidencia que indica que hasta el más experimentado en manejo de la vía aérea, podría tener problemas para determinar a qué profundidad debe quedar el tubo endotraqueal ${ }^{7-10}$.

Rutinariamente se afirma que el tubo endotraqueal debe ser fijado en promedio a $23 \mathrm{~cm}$ en hombres y $21 \mathrm{~cm}$ en mujeres, lo cual también es extrapolado de estudios con pacientes caucásicos; sin embargo, al haber una diferencia de estatura promedio entre hombres y mujeres también existe una diferencia en la longitud de la vía aérea, aunque esto no indica que el sexo sea una variable que se relacione independientemente ${ }^{10-12}$.

El objetivo del presente estudio fue describir las características de la vía aérea de la población en estudio de Cuenca-Ecuador según: distancia de incisivos superiores medios a cuerdas vocales, cartílago cricoides, carina y el punto entre el tercer y cuarto anillo traqueal contado desde la carina en sentido cefálico; cuerdas vocales a carina, tráquea, número de anillos traqueales, para correlacionar estos resultados frente a estatura y sexo.

\section{METODOLOGÍA}

Estudio descriptivo. Se recolectaron los datos desde marzo 2019 a marzo 2020, de pacientes intervenidos en los quirófanos de los hospitales "José Carrasco Arteaga" y "Vicente Corral Moscoso", de la ciudad de Cuenca, Ecuador. 
Criterios de inclusión: pacientes con edad igual o mayor de 18 años, sometidos a cirugía con anestesia general, que aceptaron participar en el estudio y firmaron el consentimiento informado. Según la clasificación del estado físico actual de la Sociedad Americana Anestesiología (Asa Ps) I, II o III. Programado para intubación con broncoscopio.

Criterios de exclusión: pacientes con alteraciones anatómicas conocidas de columna, cuello y/o vía aérea. Inestabilidad hemodinámica y/o respiratoria. Sin ayuno previo según protocolos de cada institución. Con posibilidad de aspiración de contenido gástrico. Que requieran intubación de secuencia rápida. Con cirugías previas de mentón o de cuello. Con deformidad o edema de cara, vía aérea, esternón y/o tórax.

A los participantes que cumplieron los criterios de inclusión se les solicitó su consentimiento informado que previamente fue aprobado por la Comisión de Bioética e Investigación de la Universidad de Cuenca.

Se recolectaron los datos con el mismo flexómetro, estadímetro y broncoscopio flexible Ambu Scope 3 según protocolos de bioseguridad de cada hospital, todas las medidas fueron tomadas en centímetros y se registraron en un formulario diseñado para dicho efecto. A los pacientes que aceptaron participar se les realizó una inducción anestésica según los protocolos de cada hospital y criterio del médico tratante de anestesiología.

Con el paciente en decúbito supino en una superficie recta, asegurando que la cabeza esté en posición neutra, cada medida fue tomada por el médico que realizó la broncoscopía, verificada y anotada por un ayudante de la siguiente manera:
Luego de la inducción, usando broncoscopio flexible (longitud $60 \mathrm{~cm}$ ), se midió desde la parte proximal del broncoscopio flexible a los incisivos superiores y se obtuvo las medidas restando el total, según: distancia de incisivos centrales superiores a cuerdas vocales. Distancia de incisivos centrales superiores a cartílago cricoides. Distancia de incisivos centrales superiores a carina. Distancia entre los incisivos centrales superiores a la distancia entre el tercer o cuarto anillo traqueal contado desde la carina en sentido cefálico, al finalizar se contó el número de anillos traqueales. En caso de ausencia de los incisivos centrales, se midió desde la encía.

Para obtener el tamaño de la tráquea se restó la medida de los incisivos centrales superiores a la carina menos los incisivos centrales superiores al cartílago cricoides; la medida de cuerdas vocales a la carina se obtuvo restando los incisivos centrales superiores a la carina menos incisivos centrales superiores a las cuerdas vocales.

Tras análisis con las pruebas de normalidad se utilizó estadística descriptiva y correlación de Spearman para analizar las dimensiones de la vía aérea en la población estudiada con el software SPSS versión 22.0.

\section{RESULTADOS}

Durante el estudio se evaluaron las dimensiones de la vía área en 151 pacientes, la edad promedio fue de 42.40 años, la mayoría de pacientes fueron masculinos con el $51 \%$, la altura promedio fue 1.59 metros (ver Tabla $\mathrm{N}^{\circ} 1$ ). El tiempo para intubar y tomar las medidas fue menos de 2 minutos en todos los casos, no hubo eventos adversos con secuelas.

Tabla $N^{\circ} 1$

Características de la población de estudio según edad, sexo y estatura. Hospitales "Vicente Corral Moscoso" y "José Carrasco Arteaga". Cuenca. 2019 -2020

\begin{tabular}{lcl}
\hline \multicolumn{1}{c}{ Variable } & & \\
\hline Edad en años & $\mathrm{X} \pm \mathrm{DE}$ & \\
& $42.40 \pm 16.25$ & $\begin{array}{c}\text { Mínimo 18 } \\
\text { Máximo 88 }\end{array}$ \\
Sexo & $\mathrm{n} \quad(\%)$ & \\
$\quad 77(51.00)$ & \\
Masculino & $74(49.00)$ & \\
Eemenino & $\mathrm{X} \pm \mathrm{DE}$ & \\
& $1.59 \pm(0.09)$ & Mínimo 1.40 \\
& & Máximo 1.85 \\
\hline
\end{tabular}


Se obtuvo una media de los incisivos medios a las cuerdas vocales de $13.07 \pm(1.36) \mathrm{cm}$; de los incisivos medios al cricoides un promedio de 14.93 $\pm(1.38) \mathrm{cm}$; de los incisivos medios a la carina de $23.32 \pm(1.98) \mathrm{cm}$; con un número de anillos traqueales de 13. Con estos datos se calculó el tamaño de la tráquea restando la medida de los incisivos superiores a la carina menos incisivos superiores al anillo cricoides, se obtuvo una media de $8.40 \pm(1.37) \mathrm{cm}$, con un mínimo de $6 \mathrm{~cm}$ y un máximo de $13 \mathrm{~cm}$, siendo en hombres de $9.00 \pm$ (1.16) $\mathrm{cm}$ y en mujeres de $7.75 \pm(1.39) \mathrm{cm}$ (Ver Tabla $\mathrm{N}^{\circ} 2$ ).

\section{Tabla $\mathrm{N}^{\circ} 2$}

Características de la vía aérea de la población estudiada, según promedio de total de los pacientes y por sexo. Hospitales "Vicente Corral Moscoso" y “José Carrasco Arteaga”. Cuenca. 2019 -2020

\begin{tabular}{|c|c|c|c|c|}
\hline Variable & $\begin{array}{c}\text { Total } \\
X \pm D E\end{array}$ & $\begin{array}{c}\text { Hombres } \\
X \pm D E\end{array}$ & $\begin{array}{c}\text { Mujeres } \\
X \pm D E\end{array}$ & $\mathbf{p}$ \\
\hline \multicolumn{5}{|c|}{ Incisivos medios a cuerdas vocales en $\mathrm{cm}$} \\
\hline & $13.07 \pm 1.36$ & $13.75 \pm 1.18$ & $12.37 \pm 1.17$ & 0.000 \\
\hline \multicolumn{5}{|c|}{ Incisivos medios a cricoides en $\mathrm{cm}$} \\
\hline & $14.93 \pm 1.38$ & $15.65 \pm 1.21$ & $14.19 \pm 1.13$ & 0.000 \\
\hline \multicolumn{5}{|c|}{ Número de anillos traqueales } \\
\hline & $\begin{array}{l}13.64 \pm 1.69 \\
\text { Moda } 13\end{array}$ & $\begin{array}{l}13.66 \pm 1.89 \\
\text { Moda } 13\end{array}$ & $\begin{array}{l}13.62 \pm 1.47 \\
\text { Moda } 13\end{array}$ & 0.908 \\
\hline \multicolumn{5}{|c|}{ Incisivos medios a carina en $\mathrm{cm}$} \\
\hline & $23.32 \pm 1.98$ & $24.54 \pm 1.60$ & $22.05 \pm 1.48$ & 0.000 \\
\hline \multicolumn{5}{|c|}{ Incisivos medios al espacio entre tercer y cuarto anillo traqueal en $\mathrm{cm}$} \\
\hline & $20.81 \pm 2.03$ & $21.86 \pm 1.69$ & $19.72 \pm 1.78$ & 0.000 \\
\hline \multicolumn{5}{|c|}{ Tráquea en cm } \\
\hline & $8.40 \pm 1.37$ & $8.89 \pm 1.15$ & $7.89 \pm 1.39$ & 0.000 \\
\hline \multicolumn{5}{|c|}{ Cuerdas vocales a carina en $\mathrm{cm}$} \\
\hline & $10.24 \pm 1.45$ & $10.78 \pm 1.24$ & $9.68 \pm 1.45$ & 0.000 \\
\hline
\end{tabular}

Al realizar un test de correlación de la población total, existe una moderada correlación con una $p$ de 0.000 en todas las medidas, excepto el número de anillos traqueales, pero si se divide por sexo los valores de correlación bajan y la mayoría tienen una $p>0.05$ (ver Tabla $N^{\circ} 3$ ). 


\section{Tabla $\mathrm{N}^{\circ} 3$}

Correlación según sexo de las medidas de la vía aérea de la población estudiada y estatura. Hospitales "Vicente Corral Moscoso" y "José Carrasco Arteaga”. Cuenca. 2019 -2020

\begin{tabular}{|c|c|c|c|}
\hline Variable & Total & Hombres & Mujeres \\
\hline \multicolumn{4}{|c|}{ Incisivos medios a cuerdas vocales en $\mathrm{cm}$} \\
\hline & $0.492 p=0.000$ & $0.243 p=0.033$ & $0.130 p=0.271$ \\
\hline \multicolumn{4}{|c|}{ Incisivos medios a cricoides en $\mathrm{cm}$} \\
\hline & $0.530 p=0.000$ & $0.284 p=0.012$ & $0.218 p=0.062$ \\
\hline \multicolumn{4}{|c|}{ Número de anillos traqueales } \\
\hline & $0.062 p=0.448$ & $0.182 p=0.113$ & $-0.030 p=0.798$ \\
\hline \multicolumn{4}{|c|}{ Incisivos medios a carina en $\mathrm{cm}$} \\
\hline & $0.656 p=0.000$ & $0.310 p=0.006$ & $0.340 p=0.003$ \\
\hline \multicolumn{4}{|c|}{ Incisivos medios al espacio entre tercer y cuarto anillo traqueal en $\mathrm{cm}$} \\
\hline & $0.509 p=0.000$ & $0.158 p=0.170$ & $0.183 p=0.120$ \\
\hline \multicolumn{4}{|c|}{ Tráquea en cm } \\
\hline & $0.425 p=0.000$ & $0.198 p=0.085$ & $0.179 p=0.127$ \\
\hline
\end{tabular}

* Se realiza Rho de Spearman debido a datos no normales.

En la población de estudio la medida de la tráquea fue en promedio de $8.40 \pm(1.37) \mathrm{cm}$, de los incisivos medios a la carina de $23.32 \pm(1.98) \mathrm{cm}$, de los incisivos medios al cricoides de $14.93 \pm(1.38)$ $\mathrm{cm}$, a las cuerdas vocales de $13.07 \pm(1.36) \mathrm{cm}$, al tercer o cuarto anillo traqueal desde la carina 20.81 \pm (2.03). La mayoría tuvo 13 anillos traqueales.

\section{DISCUSIÓN}

Los estudios para medir la vía aérea han sido realizados conforme el avance de la tecnología con diferentes técnicas, al inicio en cadáveres, después por medio de imágenes como tomografías y radiografías; cada método con sus limitaciones y correcciones, es por ello que actualmente por su visión directa y mínimo error, la broncoscopía flexible es una alternativa para obtener medidas de longitud, aptas para comparar con las medidas de estudios previos ${ }^{1,4,5}$

En 1982 en Estados Unidos se realizó un estudio en el cual se evaluó la longitud de la tráquea por medio de Tomografía Computarizada (TAC), obteniendo como resultados una longitud de $13.1 \pm$ $(0.9) \mathrm{cm}$ en hombres y $11.8 \pm(1) \mathrm{cm}$ en mujeres. Al comparar con los valores del presente estudio, en hombres de $9.00 \pm(1.16) \mathrm{cm}$ y en mujeres de 7.75 $\pm(1.3) \mathrm{cm}$, se evidencia que la longitud es mayor en los caucásicos ${ }^{13}$.

De igual forma, un estudio realizado en población australiana en el año 2010 en 150 pacientes, expone que la distancia de las cuerdas vocales a la carina tiene una media de $12.70 \mathrm{~cm}$ en general para ambos sexos, con un rango de $11.8 \pm 1.3 \mathrm{~cm}$ y $13.6 \pm 1.4 \mathrm{~cm}$ en mujeres y hombres respectivamente ${ }^{14}$. En el actual estudio se encontró que la distancia en hombres es de $10.78 \pm(1.24) \mathrm{cm}$ y en mujeres de $9.68 \pm(1.45)$ $\mathrm{cm}$, siendo en total de $10.24 \pm(1.45) \mathrm{cm}$.

Durante 30 años en la población sueca, se realizó mediciones de la laringe y la tráquea en material de autopsias de adultos, obteniéndose una media de 16.4 anillos traqueales en mujeres y 16.8 en hombres ${ }^{15}$. En la población de estudio la mayoría tuvo 13 anillos traqueales en ambos sexos.

En otro estudio realizado en México en 2009 en cadáveres, se demuestra que la longitud traqueal en mujeres fue de $8.6 \pm 0.5 \mathrm{~cm}$ (rango 7.8 a 9.5 ) y en hombres de $9.1 \pm 0.9 \mathrm{~cm}$ (rango 8.0 a 10.7) . $^{4}$. Se evidencia que los valores ciertamente se asemejan a la población de estudio, obteniendo una media traqueal $8.40 \pm(1.37) \mathrm{cm}$, con un mínimo de $6 \mathrm{~cm}$ y un máximo de $13 \mathrm{~cm}$, en hombres de $9.00 \pm(1.16)$ $\mathrm{cm}$ y en mujeres de $7.75 \pm(1.39) \mathrm{cm}$. 
En Colombia en el 2016, se investigó en 516 pacientes en donde la distancia bocacarina es de $20.4 \mathrm{~cm}$ en hombres y $19.2 \mathrm{~cm}$ en mujeres, desde los incisivos medios a la carina en posición neutra ${ }^{5}$. En población estadounidense la medida de los incisivos superiores a la carina fue de 28.5 $\mathrm{cm}$ en hombres y $25.2 \mathrm{~cm}$ en mujeres ${ }^{16,17}$. En la población de estudio se obtuvo una medida de $24.50 \pm(1.60) \mathrm{cm}$ en hombres y $22.00 \pm(1.48)$ $\mathrm{cm}$ en mujeres.

Al realizar la correlación de los datos de las medidas de la vía aérea con estatura, se muestra correlación baja cuando se divide por sexo, aunque hay correlación mayor moderada en el total de la población, lo que concuerda con los estudios realizados en los últimos años con resultados similares ${ }^{3,5,18}$.

Los resultados obtenidos son compatibles con estudios realizados en diferentes partes del mundo, se evidencia que la vía aérea tiene diferentes tamaños dependiendo de la etnia, debido a la altura promedio de la población estudiada; los resultados de estudios de etnias como la caucásica no deben ser extrapolados a otras poblaciones como la del presente estudio ${ }^{3-6}$. Habitualmente se toman los datos de estudios de países de población blanca, que al ser aplicados en el medio pueden provocar complicaciones y aumentar la morbilidad y mortalidad de los pacientes.

La limitación del presente estudio radica en que no se obtuvo la muestra al azar, sino por conveniencia dependiendo de las condiciones del hospital y de los investigadores.

\section{CONCLUSIONES}

La medida de la tráquea fue en promedio de $8.40 \pm$ $(1.37) \mathrm{cm}$, de los incisivos medios a la carina de 23.32 $\pm(1.98) \mathrm{cm}$, de los incisivos medios al cricoides de $14,93 \pm(1,38) \mathrm{cm}$, a las cuerdas vocales de $13.07 \pm$ $(1.36) \mathrm{cm}$, al tercer o cuarto anillos traqueal desde la carina $20.81 \pm(2.03)$. La mayoría de pacientes tuvo 13 anillos traqueales.

Las medidas de la vía aérea obtenidas están en concordancia con los estudios de poblaciones parecidas a la de estudio, registrando valores menores a la población caucásica, por tanto, extrapolar los datos de ésta población puede ocasionar errores en la intubación de pacientes locales.

\section{ASPECTOS BIOÉTICOS}

El presente trabajo se realizó bajo el consentimiento informado de los pacientes, con total confidencialidad.

\section{INFORMACIÓN DE LOS AUTORES}

- Serrano Béjar Leonardo Alfredo. Médico. Especialista en Anestesiología. Libre ejercicio. Cuenca-Azuay-Ecuador.

e-mail: lasb91@gmail.com

ORCID: https://orcid.org/0000-0002-7347-0754

- Reinoso Murillo Priscila Victoria. Médica. Especialista en Anestesiología. Libre ejercicio. Cuenca-Azuay-Ecuador.

e-mail: pichyreinoso@hotmail.com

ORCID: https://orcid.org/0000-0003-2000-8897

- Peñafiel Martínez Nadia Rosalía. Médica. Especialista en Anestesiología. Hospital Vicente Corral Moscoso. Quirófano. Anestesiología. Cuenca-Azuay-Ecuador.

e-mail: nadiarpm@hotmail.com

ORCID: https://orcid.org/0000-0002-3898-4784

- Vaca Morocho Andrea Jackeline. Médica. Ministerio de Salud Pública. Centro de salud La Merced. Medicina general. Cuenca-AzuayEcuador.

e-mail: andreavm391@gmail.com

ORCID: https://orcid.org/0000-0001-7960-965X

- Serrano Béjar Mónica Viviana. Médica. Libre ejercicio. Cuenca-Azuay-Ecuador

e-mail: monica.viviana@hotmail.com

ORCID: https://orcid.org/0000-0001-6308-9987

\section{CONTRIBUCIÓN DE LOS AUTORES}

Todos los autores contribuyeron en la concepción y diseño del trabajo, recolección de datos, redacción, revisión y modificaciones, aprobación de la versión final.

\section{CONFLICTO DE INTERESES}

Artículo basado en el trabajo previo a la obtención del título de anestesiólogo "Relación entre la ubicación del tubo endotraqueal y distancia de la protuberancia mentoniana al ángulo de Louis, Hospital Vicente Corral Moscoso, Hospital José Carrasco Arteaga. 
Cuenca. 2019-2020" disponible en el repositorio digital de la Universidad de Cuenca, http://dspace. ucuenca.edu.ec/handle/123456789/36127

\section{FUENTES DE FINANCIAMIENTO}

Autofinanciado por los autores.

\section{BIBLIOGRAFÍA}

1. Schellinger $R$. The length of the airway to the bifurcation of the trachea. Anesthesiology [Internet]. 1964 [citado 16 de mayo de 2021];25:169-72. Disponible en: https:// pubmed.ncbi.nlm.nih.gov/14156544/

2. Bissinger U, Lenz G, Kuhn W. Unrecognized endobronchial intubation of emergency patients. Ann Emerg Med [Internet]. 1 de agosto de 1989 [citado 8 de septiembre de 2018];18(8):853-5. Disponible en: http://linkinghub.elsevier.com/retrieve/pii/ S0196064489802112

3. Varshney M, Sharma K, Kumar R, Varshney PG. Appropriate depth of placement of oral endotracheal tube and its possible determinants in Indian adult patients. Indian J Anaesth [Internet]. septiembre de 2011 [citado 9 de mayo de 2021];55(5):488-93. Disponible en: https://pubmed.ncbi.nlm.nih. gov/22174466/

4. Munguía-Canales D, Ruiz-Flores J, Kosai Vargas-Mendoza G, Morales-Gómez J, Méndez-Ramírez I, Murata C. Dimensiones traqueales en población mexicana. Cirugía y Cirujanos [Internet], noviembrediciembre de 2011 [citado 9 de mayo de 2021];79(6):505-510. Disponible en: https://biblat.unam.mx/es/revista/cirugia-ycirujanos/articulo/dimensiones-traquealesen-poblacion-mexicana

5. Gómez C, Melo L, Orozco Y, Chicangana C, Osorio C. Estimación de la longitud óptima de inserción del tubo orotraqueal en adultos. Rev Colomb Anestesiol [Internet], julio-septiembre 2016 [citado 9 de mayo de 2021];44(3):230-5. Disponible en: http:// dx.doi.org/10.1016/j.rca.2016.05.001

6. García H, Valencia O, López R, Gutiérrez SE. Airway anatomy for the bronchoscopist: An anesthesia approach. Revista Colombiana de Anestesiologia [Internet], julio-septiembre 2014 [citadi ek 9 de mayo de 2021];42(3):192-8. Disponible en: http:// www.scielo.org.co/scielo.php? pid $=$ S0120$33472014000300008 \& \mathrm{script}=\mathrm{sci}$ arttext\&tlng=en

7. Cornelius B, Sakai T. Inadvertent endobronchial intubation in a patient with a short neck length. Anesthesia Progress, [Internet]. 2015 [citado 9 de mayo de 2021]; 62(2):66-70. Disponible en: https://pubmed. ncbi.nlm.nih.gov/26061576/

8. Reed D, Clinton J. Proper depth of placement of nasotracheal tubes in adults prior to radiographic confirmation. Acad Emerg Med, [Internet], diciembre de 1997 [citado 9 de mayo de 2021];4(12):1111-4. Disponible en: https://pubmed.ncbi.nlm.nih.gov/9408424/

9. Thota R, Bhandari S, Jedge P, Gaitonde K, Dewoolkar L. Endobronchial Intubation: A Method For Confirmation Of Endotracheal Intubation In Infants. The Internet Journal of Anesthesiology, [Internet], 2006 [citado 9 de mayo de 2021];13(2):1-3. Disponible en: http://ispub.com/IJA/13/2/7237

10. Caplan R, Posner K, Ward R, Cheney F. Adverse respiratory events in anesthesia: A Closed Claims Analysis. Anesthesiology [Internet]. 1990 [citado 16 de mayo de 2021];72(5):828-33. Disponible en: https:// pubmed.ncbi.nlm.nih.gov/2339799/

11. Pak H, Hong B, Lee W. Assessment of airway length of Korean adults and children for otolaryngology and ophthalmic surgery using a fiberoptic bronchoscope. Korean $\mathrm{J}$ Anesthesiol [Internet]. octubre de 2010 [citado 16 de mayo de 2021];59(4):249-55. Disponible en: https://www.ncbi.nlm.nih.gov/ pmc/articles/PMC2966705/

12. Al-Qahtani A, Messahel F, Ouda W. Inadvertent endobronchial intubation: A sentinel event. Saudi J Anaesth [Internet]. julio de 2012 [citado 16 de mayo de 2021];6(3):259-62. Disponible en: https:// pubmed.ncbi.nlm.nih.gov/23162400/

13. Cherng C, Wong C, Hsu C, Ho S. Airway length in adults: estimation of the optimal endotracheal tube length for orotracheal intubation. J Clin Anesth [Internet]. junio de 2002 [citado 16 de mayo de 2021];14(4):2714. Disponible en: https://pubmed.ncbi.nlm. nih.gov/12088810/ 
14. Griscom N. Cross-sectional shape of the child's trachea by computed tomography. Am J Roentgenol [Internet]. junio de 1983 [citado 16 de mayo de 2021];140(6):1103-6. Disponible en: https://pubmed.ncbi.nlm.nih. gov/6602473/

15. Pang G, Edwards M, Greenland K. Vocal cords-carina distance in anaesthetised Caucasian adults and its clinical implications for tracheal intubation. Anaesth Intensive Care [Internet]. noviembre de 2010 [citado 16 de mayo de 2021];38(6):1029-33. Disponible en: https://pubmed.ncbi.nlm.nih. gov/21226433/

16. Randestad A, Lindholm CE, Fabian P. Dimensions of the cricoid cartilage and the trachea. The Laryngoscope [Internet]. enero de 2009 [citado 16 de mayo de 2021];110(11):1957-61. Disponible en: https://onlinelibrary.wiley.com/doi/ full/10.1097/00005537-200011000-00036.

17. Randestad A, Lindholm CE, Fabian P. Dimensions of the cricoid cartilage and the trachea. Laryngoscope [Internet]. 1 de noviembre de 2000 [citado 16 de mayo de 2021];110(11):1957-61. Disponible en: https://onlinelibrary.wiley.com/doi/ full/10.1097/00005537-200011000-00036.

18. Ge, X., Huang, H., Bai, C. et al. The lengths of trachea and main bronchus in Chinese Shanghai population. Sci Rep 11, 2168 (2021). https://doi.org/10.1038/s41598-02181744-0. 